\title{
RETRICCIONESDELCANALDECOMUNICACIÓNEFECTOSPSICOSOCIALESEN ENTORNOSEDUCATIVOS
}

\author{
Jorge Ricardo Vivas ${ }^{1}$ \\ Nancy Noemí Terroni ${ }^{2}$ \\ Lila Ricci ${ }^{3}$
}

\section{Resumen}

\begin{abstract}
Uno de los fenómenos más notables en el campo de la educación y la capacitación ha sido el creciente interés por el aprendizaje abierto y a distancia mediado por nuevas tecnologías. Si bien resulta innegable una ganancia en cuanto a la accesibilidad de los actores al liberarlos de las limitaciones de tiempo y espacio en la interacción electrónicamente mediada, no resultan tan bien conocidas las consecuencias emergentes de las restricciones en la presencia social. En este trabajo se sugiere que como producto de propiedades intrínsecas del medio de comunicación queda afectado el acceso y distribución de la participación de los actores. Se reportan los resultados de una observación de grupos de alumnos trabajando en forma presencial y mediada por computadora en una tarea de toma de decisión denominada Moon Survival. Utilizando análisis de redes sociales se pudo establecer diferencias significativas en el nivel de actividad, la participación y la cohesión de las redes de comunicación para cada medio. La distribución de la participación de los agentes en comunicación mediada por computadora pone de manifiesto que este medio replica y magnifica las desigualdades en la utilización del recurso por parte de los diferentes actores implicados en la tarea.Palabras Claves: presencia social, participación, comunicación mediada por computadora.
\end{abstract}

\section{Channel COMmunication ReStrictions. Psychosociological EFfeCts in EduCational ENVIRONMENTS}

\begin{abstract}
The growing interest on the open and distance education mediated by new technologies is one of the most remarkable phenomenon in educational field. Although the gain as regards accessibility of the actors is undeniable when they are free of time and space limitations in electronic mediated interaction, the emergent consequences of the social presence restrictions are not so well known. This paper suggested that the access and the distribution of the actor's participation are affected as a product of intrinsic properties of the communication media. There are reported results of an observation of student groups working face to face and computer mediated in a making decision task called Moon Survival. Significant differences in the level of activity, participation and communication network cohesion, for each medium, were established by the use of social network analysis. The distribution of the agents' participation in computer mediated communication reveals that this medium increases and expands the differences in the use of the resources by the different actors who are involved in the task.Key words: social presence, participation, computer mediated communication.
\end{abstract}

\section{INTRODUCCIÓN}

El desarrollo de las nuevas tecnlogías de la información y la comunicación ha tenido un crecimiento vigoroso en el último cuarto de siglo con el consecuente impacto en el campo de la educación y la capacitación. Eso indujo un sostenido avance en la investigación y desarrollo de la tecnología educativa y la reactualización de las respectivas instituciones y programas (García Aretio \& Marín Ibáñez, 1998).

Sin duda las modalidades educativas abierta y a distancia se han ido configurando como una parte integral de los sistemas educativos y representan tendencias democratizadoras que se esfuerzan por ampliar las

\footnotetext{
${ }^{1}$ Magíster en Psicología Social orientación Organizacional. Profesor Titular de Psicología Cognitiva y Teorías del Aprendizaje al de la Facultad de Psicología de la Universidad Nacional de Mar del Plata.

${ }^{2}$ Magíster en Estadística. Profesora Asociada del área Matemática de la Facultad de Ciencias Exactas y Naturales de la Universidad Nacional de Mar del Plata.

${ }^{3}$ Magíster en Psicología Social orientación Organizacional. Becaria de Perfeccionamiento SECYT UNMDP. De la Facultad de Psicología de la Universidad Nacional de Mar del Plata.
} 
posibilidades de acceso a la educación y al aprendizaje, liberando a los alumnos de las limitaciones horarias y de residencia y ofreciendo, por lo tanto, oportunidades más flexibles para el intercambio y la socialización del conocimiento. Este tipo de aprendizaje se ha convertido en uno de los campos de más rápido crecimiento en educación, y su impacto potencial en todos los sistemas de enseñanza ha sido muy acentuado.

$\mathrm{Si}$ bien se ha producido una ganancia indiscutible en las condiciones de accesibilidad al liberar a los actores de las restricciones del tiempo y del espacio, no resultan tan bien conocidas, sin embargo, las consecuencias emergentes de las restricciones impuestas por este tipo de mediadores pedagógicos, particularmente, los fenómenos psicosociales que se derivan de la restricción de la presencia social del interlocutor. Este concepto fue acuñado para aludir al empobrecimiento en la calidad de las señales que, en ese sentido, se deriva de la naturaleza del canal utilizado (Short, Williams, \& Christie, 1976; Culnan \& Markus, 1987). Las restricciones en la percepción visual, en la recepción de las entonaciones fonéticas y, de modo más amplio, en los alcances de la comunicación paralingüística, afectan el modo en que los agentes participan y acceden en cada situación de intercambio comunicacional. Resulta por tanto de interés, conocer con mayor precisión el modo en que las restricciones impuestas por los distintos canales de comunicación afectan el acceso, participación y democraticidad de las interacciones cuando son moduladas por cada medio.

Este trabajo reporta los resultados de una observación rigurosa de grupos de estudiantes trabajando con distinto soporte comunicacional en forma cara a cara (CAC) y mediado por computadora (CMC). Por medio de un diseño cuasi experimental, se registró la variación en la participación, en base al volumen y distribución de las alocuciones de los alumnos, y se calculó las propiedades reticulares emergentes de aplicar Análisis de Redes Sociales para comprender estructuralmente el flujo comunicacional en cada medio, mientras se desarrollaba una tarea grupal concreta, en modalidad presencial interactuando cara a cara o mediado por computadora utilizando IRC (Internet Relay Chat) por medio de un canal privado de comunicación electrónica.

Algunos antecedentes de investigaciones que vinculan el medio utilizado con la participación en diversas organizaciones.
Una parte sustantiva de la investigación psicosocial se ha concentrado en las consecuencias del filtrado de las claves no verbales en la comunicación electrónica (Sproull \& Kiesler, 1991; Zornoza, 1992; Hiltz \& Turoff, 1993). La mediación reduce la presencia social como producto de las restricciones en el contacto visual, los gestos, las señales de aprobación o los silencios y dudas antes de contestar. En la comunicación electrónica no se puede percibir ninguna señal contextual, los participantes no pueden ver la distribución de las sillas para identificar virtuales uniones y rupturas entre los compañeros, ni ubicar sitios de encuadre que permitan identificar la importancia de las reuniones ni el rol que juegan los diferentes actores. Se ha descrito que la ausencia relativa de estas señales desarrolla un lenguaje desinhibido, gestión negativa de conflicto, dificultades en la coordinación y la retroalimentación, problemas para alcanzar consenso y polarización del grupo (Kiesler, Siegel, \& Mc Guire, 1984.; Hiltz, Johnson, \& Turoff, 1986; Harasim \& Winkelmans, 1990; Kiesler \& Sproull, 1991; Peiró, Prieto \& Zornoza, 1993).

Un aspecto importante de considerar es las variaciones en la secuencialidad asociadas al canal. En los grupos comunicación CAC, la distribución de la palabra es secuencial o, cuando así no sucede, produce y manifiesta confusión. Debido a esa necesidad de coordinación, los grupos comunicación CAC se conforman típicamente con menos de una docena de miembros. Si poseen más integrantes, naturalmente se subdividen o, en ciertos casos, se produce el efecto de que algunos miembros se tornen más pasivos. Por el contrario, en el chat existen menos restricciones por su naturaleza y esto permite que las personas puedan expresar sus ideas al mismo tiempo. Sin embargo, los mensajes entran en la pantalla con una secuencialidad que no respeta las necesidades lógicas del discurso sino la velocidad motriz de los participantes y la diferente capacidad de transferencia de los dispositivos mediadores, exigiendo por lo tanto, una tarea adicional de los participantes, un esfuerzo cognitivo por reconstruir la coherencia polisintagmática que emerge de la interacción.

Otros autores establecen una comparación entre los diferentes medios de comunicación de acuerdo a la riqueza de la presencia social permitida. Daft, Lengel e Trevino (1987) afirman que mientras que los medios de mayor riqueza contribuirían a que los participantes se entiendan los unos a los otros a través de la retroalimentación, las señales múltiples, la variedad del 
lenguaje y el enfoque personal, incluida la emoción y los sentimientos; los medios de menor riqueza utilizarían fundamentalmente reglas, formas y procedimientos. Esta limitación afecta la percepción de influencia social ejercida, la que se ve alterada en la comunicación mediada por computadora al opacar el reconocimiento de las claves de influencia y exaltar el peso de la participación (Vivas \& Terroni, 2001a).

La comunicación electrónica impone restricciones de canal que filtran la presencia social del interlocutor. Esto redunda en la menor disponibilidad de claves sobre la interacción que las que existen en la comunicación cara a cara. Esta igualación parece generar menos reconocimiento de la especificidad de la participación de los miembros del grupo. Las personas se tornan más desinhibidas, no conformistas y conflictivas al usar comunicación electrónica. Es más difícil interpretar las intenciones del remitente, es más probable que surjan equívocos y más complejo resolverlos. Aunque los grupos se polarizan y tardan más en desarrollar líderes y consenso, su mayor rango de ideas puede producir decisiones más innovadoras y mejores. A la fecha, sin embargo, el enfoque de investigación estricto en toma de decisión no ha considerado integralmente en sus diseños a los estudios que tengan en cuenta relaciones anteriores entre los miembros del grupo, que analice interacciones por períodos más largos y que ocurran en organizaciones educativas reales, donde las personas deben simultáneamente asistir a una variedad de las tareas y redes sociales (Vivas \& Terroni, 2001b)

El presente trabajo explora la democraticidad que ofrece el medio en términos de acceso y distribución de la participación, para grupos de compañeros de estudio en una situación cuasi experimental de resolución conjunta de una tarea. El problema que aborda es analizar cómo las restricciones de intercambio de las claves sociales derivadas del filtro impuesto por la comunicación electrónica, afectan el monto de participación de los alumnos y a la distribución de esa participación.

De acuerdo con la revisión de la literatura existente y estudios previos llevados a cabo, se proponen las siguientes hipótesis:

1. La actividad, participación y presión comunicacional de los participantes, será mayor en comunicación mediada (CMC) que en comunicación presencial (CAC).

2. La distribución de la participación será semejante para ambos medio de comunicación

\section{MÉTODO}

\section{Participantes}

Participaron 100 alumnos de 3er. año de la carrera de Psicología de la U.N.M.D.P., cursantes regulares de las asignaturas Teorías del Aprendizaje, Psicología Cognitiva y Psicología Educacional. Los grupos se compusieron de cinco miembros. Procedimiento

Se utilizó una prueba de decisión múltiple colaborativa denominada Supervivencia en la Luna de Hall e Watson (1970). Para su selección se consideró que por su naturaleza es un ejercicio cognitivamente atractivo, adecuado al nivel de competencia intelectual de los participantes y que, como problema, se ubica como tarea decisional de tipo intelectiva, de acuerdo a los criterios de demostrabilidad de Laughlin e Ellis (1986), lo cual disminuye el peso de la asertividad del discurso y estimula la participación argumentativa. Tras un primer momento de resolución individual del problema se conforman al azar grupos de cinco miembros que en forma colectiva producen un nuevo ordenamiento, consensuando las propuestas individuales. En una tercera fase se solicita la ordenación definitiva individual. El problema tiene una solución óptima definida por un panel de expertos de la NASA que se entrega como clave de corrección a los participantes, como así también una guía de los cálculos elementales que se pueden realizar sobre la producción individual y la del pequeño grupo.

El mismo procedimiento se utilizó en la modalidad mediada, solo que para el agrupamiento e interacción electrónica en grupos de cinco, se instaló un canal privado de chat utilizando MIRC con cinco máquinas horizontalmente interconectadas y una sexta máquina de comando y registro de la actividad de la red ubicada en otra dependencia. Los participantes podían tanto interactuar en forma conjunta con los otros cuatro miembros del grupo como tener intercambios privados de pares no visibles para los otros tres integrantes.

\section{Materiales}

Se confeccionaron hojas de registro de la dinámica grupal para la modalidad cara a cara, donde se consigna la frecuencia, dirección y sentido de la comunicación entre los miembros del grupo. En la modalidad mediada se dispone también del registro de los movimientos 
conservados en la un archivo de log.

En el caso de la comunicación mediada por computadora se transcribió el proceso interaccional en un registro de observación de la prueba, el que fue volcado en matrices para su tratamiento. Este proceso, sin embargo, requirió de una actividad adicional para su consecución. Primeramente se codificó la intencionalidad comunicativa de acuerdo a los criterios de Bales (1959) de modo de emparejar los criterios con los utilizados con los observadores de los grupos cara a cara. Cada registro de comunicación electrónica fue codificado por tres jueces docentes, tres jueces alumnos universitarios adscriptos a la investigación y cuatro jueces adolescentes de entre 12 y 18 años. La validez de los resultados interjueces resultó altamente significativa $(r=0.93)$.

\section{Resultados}

Con el objeto de analizar la incidencia de la participación (comunicacional) ejercida durante la tarea, se analizó el volumen global del flujo de comunicaciones para ambas modalidades. Dado que los datos no presentaban una distribución normal (ver gráficos 1 y 2) se usó para estas comparaciones una prueba no paramétrica. Los resultados de la misma pueden verse en la tabla 1.

Como se puede observar la mediana de la cantidad de mensajes emitidos y recibidos en la modalidad CMC duplica holgadamente a los de la modalidad CAC. El nivel de actividad de las redes CMC, medida por la participación de sus miembros, es muy superior al de las redes CAC.

Es posible obtener información complementaria a la cantidad global de mensajes de entrada y salida, si comparamos, para cada miembro individual, el nivel de actividad normalizado por medio de la centralidad y prestigio y la densidad de su red personal. Los resultados permiten apreciar las diferencias entre los miembros más centrales y más periféricos de la red para cada modalidad, así como la densidad del entramado de los vínculos personales para individuo en cada grupo. Nuevamente, los datos no presentaron distribución normal por lo que fueron comparados usando la prueba no paramétrica de Mann-Whitney, resul-

I apıa I. Lomparacion de ıa cantıaad de mensajes emitıos y recioıdos entre
modalidades.

\begin{tabular}{|c|c|c|c|c|c|c|c|}
\hline Mensaje & $\mathrm{CA}$ & 50 & 133,0 & Prim & Segund & U & Signific \\
\hline emitido & $6 \mathrm{E}$ & 150 & V1, & equef: 0 & ocgă & Vlanhe 0 & clón \\
\hline ßMensaje & $6 \mathrm{~A}$ & 50 & a $q_{36,0}$ & $\log _{5,0}$ & ${ }^{\prime} \varphi_{71,2}$ & Whitney ${ }_{363,0}$ & \\
\hline $\begin{array}{l}\text { secibido } \\
\mathrm{s}\end{array}$ & $\begin{array}{l}\text { EM } \\
\text { C }\end{array}$ & 50 & $\begin{array}{l}0 \\
322,0 \\
0\end{array}$ & $\begin{array}{l}0 \\
189,5 \\
0\end{array}$ & $\begin{array}{l}5 \\
493,5 \\
0\end{array}$ & 0 & 0 \\
\hline
\end{tabular}

Gráfico 1. Mensajes emitidos por modalidad
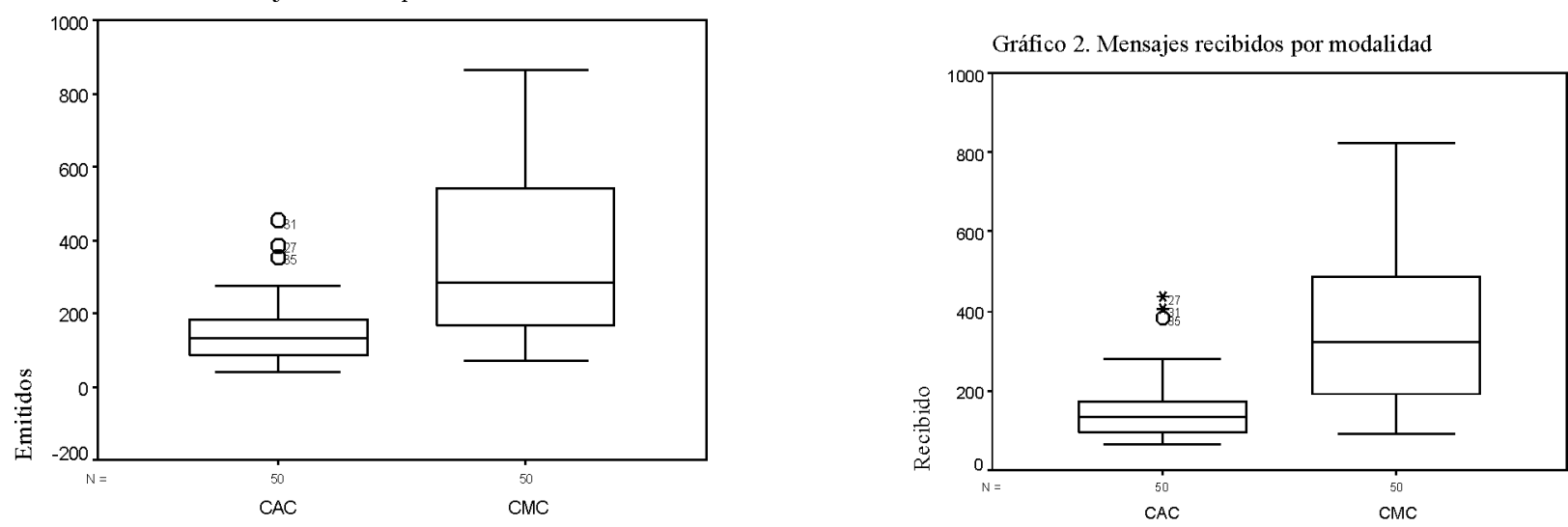

Modalidad 
tando significativamente distintos ambos medios, como se puede apreciar en la tabla 2.

Para visualizar el comportamiento de la distribución en el uso de la palabra para ambas condiciones se centralidad global para la modalidad CMC en relación a la modalidad CAC. Esas medidas, por la naturaleza de su cálculo, representan la distribución de la participación de los integrantes en la red. Una

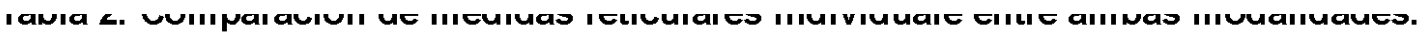

\begin{tabular}{|c|c|c|c|c|c|c|c|}
\hline & IVumaunay & Ly & jyculaua & 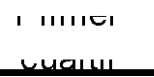 & veyuilus & $\begin{array}{c}\cup \text { uE } \\
\text { guam-y }\end{array}$ & 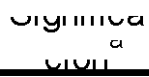 \\
\hline vall alluau & $\begin{array}{l}\text { Uחレ } \\
\text { บIVIU }\end{array}$ & u & $\begin{array}{l}\cup \cup<\cup, \cup \cup \\
, \cup<\cup, \cup \cup\end{array}$ & $\begin{array}{l}<\cup ı, \cup \cup \\
+\quad I \cup, \cup \cup\end{array}$ & $\begin{array}{l}\Psi \cup<\cup, \cup v \\
I \cup \cup ו \cup, \cup v\end{array}$ & +יט, U & ৩.৩৩৩ \\
\hline I I0oliyiu & $\begin{array}{l}\cup r \cup \\
\text { ソIVIU }\end{array}$ & $\begin{array}{l}\cup \cup \\
\cup \cup\end{array}$ & $\begin{array}{l}\cup \cup \cup, \cup \cup \\
\cup \cup \cup \cup, \cup \cup\end{array}$ & $\begin{array}{l}<\cup, \cup \cup \\
+\iota, \cup \cup\end{array}$ & $\begin{array}{l}+\angle \cup I, \angle \cup \\
I<\cup \cup I, \cup \cup\end{array}$ & $\cup \cup \cup \cup \cup$ & บ. vUv \\
\hline LCISIUau & 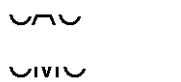 & u & 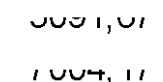 & 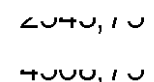 & 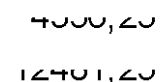 & $\cup<1, \cup \cup$ & บ. vuv \\
\hline
\end{tabular}

a.

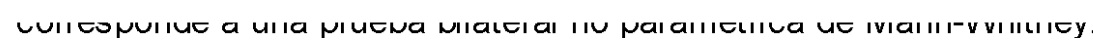

compararon a continuación, como queda expresado sintéticamente en la tabla 3 , las propiedades reticulares globales. Ello permite analizar el comportamiento de cada grupo dentro de la modalidad y las diferencias entre las mismas. Se utilizaron los siguientes índices reticulares: índice de centralidad de salida de la red (NTCOUT), índice de centralidad de entrada de la red (NTCIN) y la densidad de la red considerada como un todo (NTD).

La tabla 3 muestra que existen diferencias significativas al comparar las medianas de la centralidad global para ambas modalidades. También indica que esa asimetría se expresa en valores muy elevados de la participación igualitaria de todos los miembros de cada grupo de cinco quedaría representada por el valor cero, siendo los valores positivos que se observan en la tabla 3 indicativos de la distancia que separa al individuo más central del resto de sus compañeros de tarea para cada grupo. No es tan marcada la diferencia en el prestigio o centralidad global de entrada. Cabe señalar para la mejor interpretación de este dato, que cada vez que una persona emite un mensaje son cuatro las que se hallan recibiendo. Por lo que las propiedades obtenidas tomando como base las entradas y no las emisiones, producen una ponderación de los resultados achatando la diferencia entre los miembros.

modalidades.

\begin{tabular}{|c|c|c|c|c|c|c|c|}
\hline Centralida & CAC & & & Prime & Segund & $U$ de & Signific \\
\hline $\begin{array}{l}\text { ANTCOO } \\
\text { Prestigi }\end{array}$ & $\begin{array}{l}\mathrm{CM} \\
\epsilon_{A C}\end{array}$ & $\begin{array}{l}40 \\
10\end{array}$ & $\begin{array}{l}\text { 16estą, } 0 \\
a_{9.621,0}\end{array}$ & 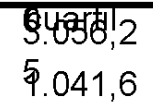 & $\begin{array}{l}\text { 96.258, } \\
33.275,0\end{array}$ & $\begin{array}{l}\text { Tann- } \\
\text { Whitney } \\
19,00\end{array}$ & $\begin{array}{l}\text { aclón } \\
, 052\end{array}$ \\
\hline $\begin{array}{l}\mathrm{PNTCl} \\
\mathrm{N})\end{array}$ & $\begin{array}{l}\mathrm{CM} \\
\mathrm{C}\end{array}$ & 10 & $\begin{array}{l}0 \\
3.362,5 \\
0\end{array}$ & $\begin{array}{l}7 \\
2.747,9 \\
2\end{array}$ & $\begin{array}{l}0 \\
5.131,2 \\
5\end{array}$ & & \\
\hline Densida & CAC & 10 & 31950,0 & 25450,0 & 45950,0 & 13,00 &, 004 \\
\hline PNTD & $\begin{array}{l}\mathrm{CM} \\
\mathrm{C}\end{array}$ & 10 & $\begin{array}{l}9_{5950,0} \\
0\end{array}$ & $\begin{array}{l}\$ 9150,0 \\
0\end{array}$ & $\begin{array}{l}931100, \\
0\end{array}$ & & \\
\hline
\end{tabular}


En la tabla 3 puede verse que la comunicación mediada por computadora es la modalidad que presenta el mayor flujo comunicacional y los valores más elevados de cohesión. La densidad de la red considerada como un todo se expresa por el cociente entre los vínculos realmente existentes y todos los vínculos posibles. En este caso, la modalidad CMC se caracteriza por una densidad global significativamente mayor respecto a la modalidad presencial.

Los gráficos 3, 4 y 5 resultan muy ilustrativos del comportamiento de esas variables. La emisión y recepción de mensajes es muy superior en la modalidad CMC. Sin embargo, podemos observar al mismo tiempo que la variabilidad del rango global de emisiones y de recepciones en ese medio es mucho más amplia que en la modalidad comunicación CAC.
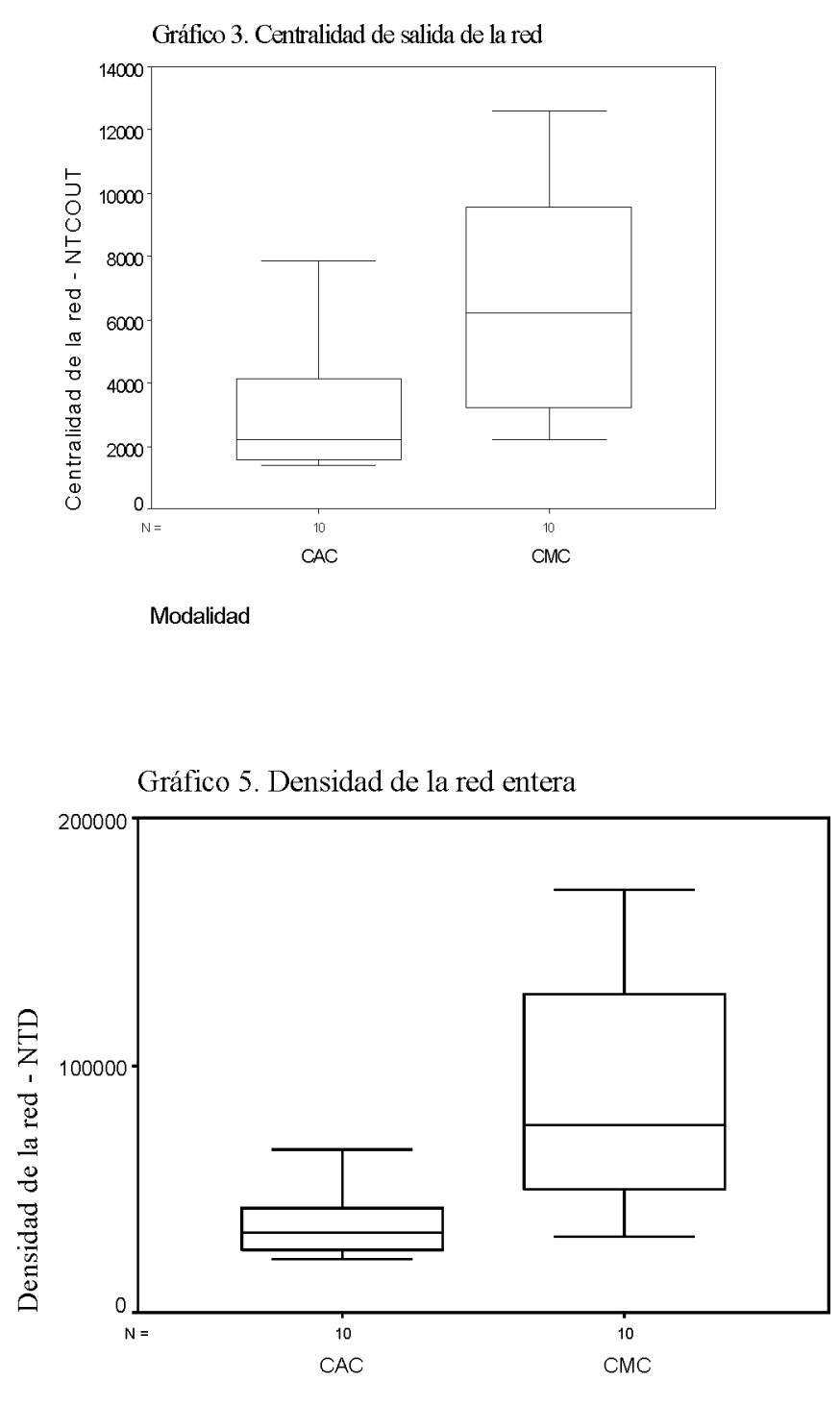

Modalidad
Los gráficos 3 y 4 ilustran los resultados de la tabla 3: la medida en que la centralidad de entrada de la red para ambas modalidades presenta un rango menor que el de salida. Eso es producto, como se ha señalado, de considerar que cada emisor, en principio, afecta a cuatro receptores - salvo que se encuentre realizando una comunicación privada - lo que disminuye la carga de su participación al distribuirla de tal modo.

La densidad del entramado comunicacional de la red considerada como un todo, que representa la cohesión global del grupo y la presión comunicacional que ejerce sobre los miembros, puede analizarse visualmente en el gráfico 5.

Las diferencias en la cohesión reticular para ambas modalidades resulta claramente apreciable, con valo-

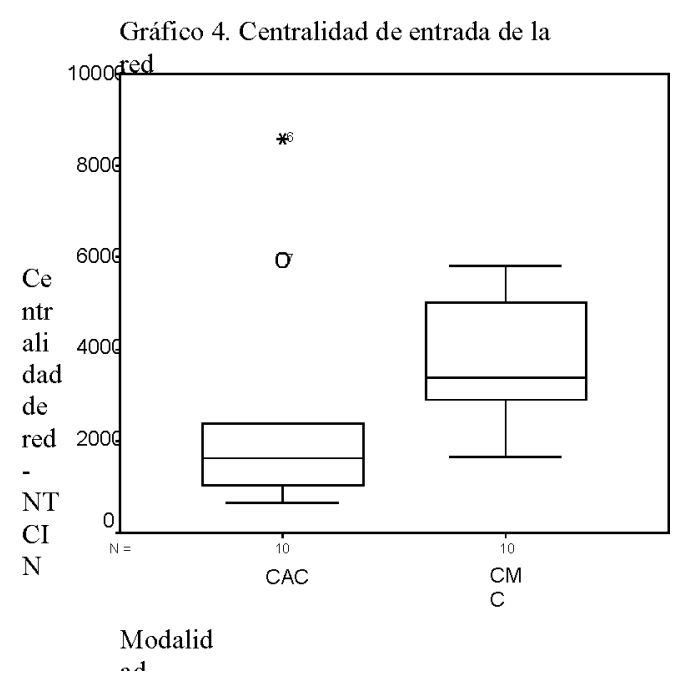

res en $\mathrm{CMC}$ que en su punto más bajo son superiores a los más altos que pueden observarse en CAC. El rango de variación de la densidad, al mismo tiempo, tiene en comunicación mediada una amplitud marcadamente superior al que se observa en comunicación cara a cara.

Estos resultados indicaron que existe una variación muy fuerte de la varianza para cada modalidad. Los resultados de esta comparación se pueden apreciar con absoluta claridad los gráficos 6 y 7 .

Tanto en uno como en otro caso esta comparación refleja las diferencias de la distribución del uso de la palabra para cada una de las modalidades estudiadas. 

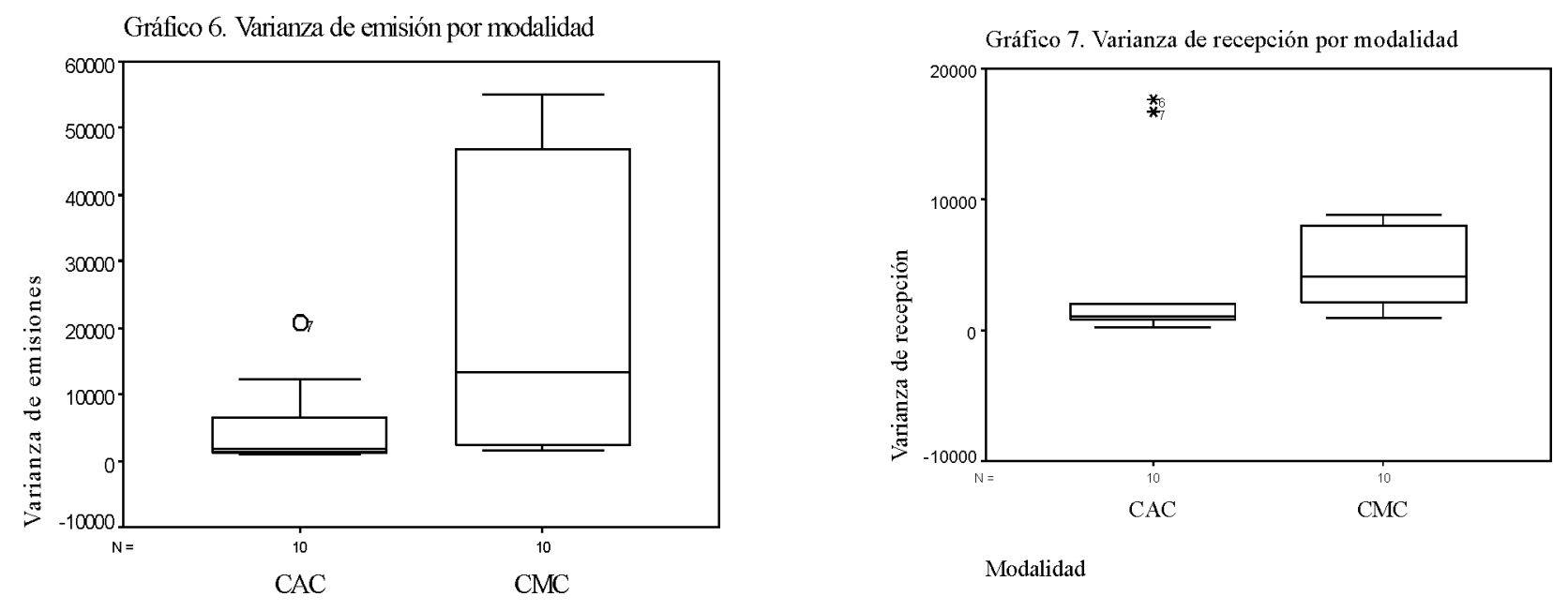

Modalidad

\section{Discusióny CONCLUSIONES}

\section{Análisis de las propiedades globales}

El primer análisis pone de manifiesto que la diferencia en el flujo global de mensajes, tratados en forma bruta, es estadísticamente significativa a favor de la modalidad CMC, con un volumen que duplica, holgadamente, la cantidad de mensajes intercambiados en la condición comunicación CAC. Estos resultados constituyen un indicador indiscutible de la mayor participación y actividad en la modalidad mediada.

Si bien la precisión de las causas de ésta mayor actividad requiere de un análisis cualitativo complementario del contenido de estos intercambios, de modo general el incremento del flujo comunicacional parece responder a un conjunto de factores que interactúan entre sí, de los cuales, en este trabajo destacamos dos grupos de fenómenos. Por un lado, los atinentes al tipo de procesamiento cognitivo que cada modalidad impone a los participantes y por otro, los que resultan emergentes del filtrado de señales impuestos por el nivel de restricción inherente a la naturaleza del canal utilizado.

En el primer grupo se destaca que el procesamiento cognitivo utilizado para la percepción y ejecución del acto de habla es más veloz que el requerido para el procesamiento de las actividades de lectoescritura. El trabajo en IRC se caracteriza por propiedades que son intrínsecas a la modalidad y que afectan de modo insoslayable la actividad de los participantes. Por un lado, es una modalidad de comunicación sincrónica (todos los participantes pueden emitir y recibir al mismo tiempo) pero, por otro, la forma de emisión es la escritura y la forma de recepción es la lectura. Estas actividades requieren habilidades cognitivas que tienen una velocidad de ejecución restringida por la naturaleza de los procesos implicados y que, en todos los casos, son más y más variados que los que intervienen en el procesamiento del habla, tal como se presenta en la modalidad comunicación cara a cara.

Entre los fenómenos comunicacionales derivados de la restricción de la presencia social (Short \& cols, 1976) que en esta tarea contribuyeron a elevar el nivel de actividad en la comunicación mediada, merecen destacarse los siguientes:

Marchas y contramarchas argumentativas que se reflejan en el registro de las intervenciones de los integrantes del grupo. Aclaraciones y correcciones permanentes en el discurso, producidas por la frecuente ruptura de la secuencialidad de los mensajes entre los participantes. Estos fenómenos ponen de manifiesto las dificultades, por parte de los participantes, para reconocer al destinatario de la intención comunicativa del emisor. Los criterios basados en comunicación paraverbal propuestos por Bales (1959) con el fin de determinar el blanco de la intención comunicativa, no resultan adecuados y son insuficientes para la comunicación mediada.

Aclaraciones y ampliaciones argumentativas recurrentes, con cierta insistencia para expresar una opinión cuando ésta no es seguida de una confirmación textual más o menos inmediata de parte de los recep- 
tores. Estas actitudes, probablemente, puedan ser interpretadas como producto de la declinación de la presencia social, con efectos como la ausencia de elementos paralingüísticos que confirmen, complementen, contextúen y orienten el sentido de un argumento y las señales de aceptación o de rechazo por parte del grupo de trabajo.

La restricción de la presencia social que circunscribe la actividad a la exposición argumentativa escrita en CMC, provoca que los participantes se encuentran frecuentemente con severas limitaciones para expresar su convicción, defender sus puntos de vista y remarcar los distintos valores otorgados a cada implicación semántica. En este caso frecuentemente los integrantes apelaron a recursos gráficos complementarios como la utilización de mayúsculas, signos de admiración o repetición textual para remarcar el sentido.

De este modo, se confirmaron las ideas previas que configuraron la primera hipótesis establecida en este trabajo. La comunicación mediada por la computadora, estudiada por medio de su estructura reticular, presenta un mayor nivel de actividad y participación que la que se puede apreciar en la comunicación cara a cara. Presumiblemente ese fenómeno sea resultante del filtrado de claves sociales que induce a una restricción de la presencia social, aunque también interviene en un grado no desdeñable la imposición de las restricciones cognitivas que impone la modalidad mediada por la lectoescritura. Desde el punto de vista pedagógico este mayor esfuerzo cognitivo de los actores, que se manifiesta como mayor actividad, no parece tener relación con un tratamiento más profundo del contenido, sino con intentos de comprender y reconstruir cognitivamente el flujo de las intenciones comunicacionales de los participantes y sus destinatarios. La mayor participación podría no redundar en una mejora de los aprendizajes sino ser el reflejo de un esfuerzo cognitivo por atribuir adecuadamente el contenido de los estados mentales de los participantes.

En los estudios canónicos de redes sociales se sugiere que existe un cierto grado de correspondencia entre la morfología reticular, la naturaleza de la tarea y algunos rasgos particulares de la participación de los integrantes de un grupo. En este caso, como el interés se centró en el estudio de los efectos de la presión social y de la cohesión del grupo durante la tarea, el análisis de las propiedades reticulares globales se concentró en las medidas de centralidad de entrada y de salida de la red entera y en la densidad de la red global. Todas las medidas pusieron de relieve diferencias significativas en los valores para ambas modalidades.

Mientras que la centralidad de un actor queda definida por su nivel de actividad, en el sentido de que el actor más activo es el que tiene más vínculos con la mayoría de los otros actores de la red, la centralidad global del grupo cuantifica la variabilidad de los índices individuales de los miembros. El índice de centralidad global expresa el rango en el cual varían los índices de centralidad de cada miembro y se considera, para una relación direccional como la comunicación, los índices de entrada y salida como recepciones y emisiones, respectivamente.

Los resultados de este tratamiento complementan los análisis anteriores. Podemos agregar ahora que, si bien el volumen de actividad global del flujo de comunicaciones y la participación individual crecen sustantivamente en comunicación mediada, la distribución de esa participación al interior de cada modalidad muestra una dispersión mucho más amplia en comunicación mediada por computadora que en comunicación cara a cara. Esto quiere decir que si bien el medio invita a una mayor fluencia comunicacional, es más sensible a la variación de la magnitud de participación individual que la comunicación presencial. Esto es, mientras en promedio general los individuos en este medio participan más, algunos actores participan mucho más. Tal vez sea acertado predicar que la comunicación mediada por computadora devuelve amplificados, en este sentido, los sesgos de participación conocidos para la comunicación presencial.

La densidad de un grafo es, tal vez, la medida más ampliamente utilizada y más recomendada para obtener la mensura del nivel de cohesión de un grupo. La cohesión comunicacional, a su vez, se considera un indicador confiable de la influencia y la presión comunicacional. Los resultados obtenidos indican claramente diferencias significativas de cohesión a favor de la comunicación mediada. Los valores en este sentido son tan amplios que se encuentran próximos a saturar la máxima cohesión posible esperada para este tipo de medida (0.87 en un rango 0-1)

En términos generales se interpreta que las redes de comunicación más cohesionadas, comparten entre sí creencias, opiniones y recursos de una manera más fluida que las más distribuidas. Sin embargo, esta 
interpretación que es adecuada para redes que se configuran espontáneamente y una relativa persistencia en el tiempo, no parece aceptable al extrapolarla a grupos artificiales que se desvanecen tras la tarea. Una interpretación más restringida de la cohesión parece resultar más plausible en este caso. De este modo se sugiere que la cohesión, en tanto medida de proximidad y presión comunicacional, es un buen indicador de la influencia ejercida por el grupo sobre sus miembros. En este sentido la cohesión de la matriz comunicacional en los grupos CMC puede ser interpretada como una medida de la influencia recibida por los miembros del grupo exclusivamente durante la realización de la tarea.

En resumen, en un contexto de aprendizaje donde la actividad requiera la puesta en común de perspectivas divergentes sobre la base de la argumentación y la

\section{REFERENCIAS}

Bales, R.F. (1959). Small group theory and research. En R.K. Merton, L.Broom \& L.S.

Cottrell, Jr. (Eds. Working papers in the theory of action. Glencoe, IL: Free Press, 293- 305.

Culnan, M.J. \& Markus, M.L. (1987). Information technologies. En F.M. Habling; L.L.

Putnam; K.H. Roberts \& L.W. Porter (Eds). Handbook of organizational communication. Newbury Park, Sage.

Daft, R.L., Lengel, R.H \& Trevino, L.K. (1987). Message equivocality media selection and manager performance: Implications for information systems. MIS Quarterly, 335-366.

García Aretio, L. \& Marín Ibañez, L. (1998) Aprendizaje Abierto y a Distancia: Perspectivas y Consideraciones Políticas. Documento de la UNESCO, UNED, España. Goode, J., \& Johnson, M. (1991). Putting out the flames: The etiquette and law of e-mail. Online, nov, 61-65.

Hall, J. \& Watson, P. (1970) The effects of a normative intervention on group Decision-Making performance. Human Relations, 23, 4, 299-317. construcción de consensos, podemos esperar que en comunicación mediada la resolución requiera más tiempo, más mensajes, más participación y, en general, mayor dificultad para sortear los obstáculos comunicacionales para lograr consensos. De acuerdo a las características del medio y a las restricciones que el mismo impone, debería considerarse cuidadosamente en entornos de aprendizajes colaborativos, el diseño de situaciones interaccionales que optimicen la calidad de las interacciones en función de los objetivos pedagógicos planteados. Desde la perspectiva de la teoría del procesamiento de la información social, argumentamos aquí que estos fenómenos psicosociales resultan ser producto del filtrado de claves sociales, como también producto de las restricciones cognitivas que impone la modalidad.

Harasim, L. M., \& Winkelmans, T. (1990). CMC scholarly collaboration. Knowledge:

creation, diffusion, utilization, 11(4), 382-409.

Hiltz, S.R., Johnson, K. \& Turoff, M. (1986). Experiments in Group Decision making: Communication Process and Outcome in face to face versus Computerized Conferences. Human Communication Research, 13, 225-252.

Hiltz, S.R. \& Turoff, M. (1993). The Network Nation. (sec. ed.) Cambridge, MA: MIT Press.

Kiesler, S., Siegel, J., \& McGuire, T.W. (1984). Social Psychological Aspects of

Computer Mediated Communication, American Psychologist, 39, 1123-34.

Kiesler, S., \& Sproull, L. (1991). Group decision making and communication technology. Organization, Behaviour and Human Decision Processes, 52, 96-123.

Laughlin, P.R.\& Ellis, A. (1986). Demonstrability and social combination processes on mathematical intellective tasks. Journal of Experimental Social Psychology, 22, 177- 189 .

Peiró, J.M., Prieto, M. \& Zornoza, A.M. (1993). Nuevas tecnologías telemáticas y trabajo grupal. Una perspectiva psicosocial. Psicothema, 5, 287-305. 
Short, J.; Williams, E. \& Christie, B. (1976). The social psychology of telecommunications. New York: John Wiley and Sons.

Sproull, L., \& Kiesler, S. (1991). Connections: New ways of working in the networked organization. Cambridge, MA: MIT Press.

Vivas, J.\& Terroni, N. (2001a). Restricciones en el canal de comunicación y representación de influencia en situación de toma de decisión. Psico-USF. 6 (1) 19-29.

Vivas, J.; Terroni, N. (2001b). Una Revisión de los estudios sobre el impacto psicosocial del correo electrónico en las organizaciones. Revista del Instituto de Rosario de Investigaciones en Ciencias de la Educación (IRICE), 15,.83-98.

Zonoza, A. (1992). La toma de decisiones en grupo. Efectos diferenciales del canal de comunicación sobre el rendimiento grupal. Tesis doctoral, octubre Valencia.

Recebido em: 02/05/02

Revisado em:06/06/02

Aprovado em:24/06/02 